\title{
Assessment of Childcare Beliefs and Practices in Tribal Population
}

\author{
M. Rathi* \\ Almas College of Nursing, Puthur Post, Kottakkal, Malappuram-676503, Kerala, India \\ *Corresponding author
}

\section{A B S T R A C T}

\section{Keywords}

Assessment, Childcare, Beliefs and practices, Tribes, Focus Group Discussion, Semi Structured Interview, Structured Direct Observation

Article Info

Accepted:

12 February 2019

Available Online: 10 March 2019
A detailed inquiry was conducted on identifying methods to assess childcare beliefs and practices in tribal population with a view to prepare data collection tool for research study entitled "Assessment of infant care practices, conduction and effectiveness evaluation of need based awareness program on infant care among mothers of selected tribal groups." Taking care of children depends on a variety of factors: the geographical location, religion and culture, socioeconomic status, educational background and the beliefs and values held by the family and its community. The tribal population, isolated geographically and socially is assumed to have distinct lifestyle practices, including childcare. Focus Group Discussion Semi Structured Interview and Structured Direct Observation are identified to be the best methods to assess their child care practices. This assessment in turn will throw light on selecting interventions to promote child health and productivity.

\section{Introduction}

"Let us be the ones who say we do not accept that a child dies every three seconds simply because he does not have the drugs that you and I had. Let us be the ones to say we are not satisfied that your place of birth determines your right to life. Let us be out raged. Let us be loud. Let us be bold."

- Brad Pitt.

Children are the resource of any country the development of a country depends on the ways it invest for the health of its childhood population. Investments lies not only in earmarking funds for the health and welfare of its children but also in developing policies and strategies for building favorable attitude towards childcare practices by their care takers.

Taking care of children, in turn, depends to a large extent on a variety of factors: the geographical location, religion and culture, socioeconomic status, educational background and the beliefs and values held by the family and its community. 
Beliefs drive us. We act the way we do due to our beliefs to a large extent. In short, our belief dictates our action and our practices including childcare practices.

The tribal population, the so called 'natives of the land' is the marginalized section of the modern society, isolated not only geographically but in all other respects. With less exposure to the outer world and civilization, their practices including those of child care are primitive and nonscientific and not amenable to change due to non-entry of external forces in to their community (Table $1)$.

Tribal development is now gaining impetus thanks to the falling parameters of health and development. An in depth study in to the beliefs and practices in child care in the tribal population will throw light on the prevailing scenario, help us to appreciate healthy practices and enable policy makers and researches to identify means to main stream them and offer a new lease of life.

Assessment is the act of judging or deciding the amount, value, quality or importance of something or the judgment or decision that is made about something: a person or situation or event. Being the marginalized section, tribal groups give an unwelcoming reception to any 'outsider' and gaining entry into and assessing their life styles is indeed a challenging task. This paper explores the assessment of childcare beliefs and practices in tribal population.

\section{Relevance}

India is the second largest country after South Africa, having a large population of Scheduled Tribes. It is fascinating that about a half of the indigenous people of the world, i.e. $84,326,240$ is living in India as per 2001 census, which constituted $8.2 \%$ of the total population of India. There were about 635 tribal groups and subgroups including 75 primitive communities who have been designated as 'primitive' based on preagricultural level of technology, low level of literacy, stagnant or diminishing population size, relative seclusion (isolation)from the main stream of population, economic and educational backwardness, extreme poverty, dwelling in remote inaccessible hilly terrains, maintenance of constant touch with the natural environment, and unaffected by the developmental process undergoing in India. There is a consensus that these scheduled tribes are the descendants of aboriginal population in India (Bhasin and Walter, 2001).

Thus we can understand that tribal groups are found all over India. The state of Orissa occupies a unique position in the tribal map of India having 62 scheduled tribes including 13 primitive tribes with a population of over 8.15 million constituting $22.3 \%$ of the population of the state as per 2001 Census.

The primitive tribes in India have distinct health problems, mainly governed by multidimensional factors such as habitat, difficult terrains, varied ecological niches, illiteracy, poverty, isolation, superstitions and deforestation. The tribal people in India have their own life styles, food habits, beliefs, traditions and socio-cultural activities. The health and nutritional problems of the vast tribal populations are varied because of bewildering diversity in their socio-economic, cultural and ecological settings (Balgir2000a). However, data analysis in view of their ecological, ethnological, cultural and biological diversity is lacking in India.

From table 2, we infer that apart from the high mortality indicators there is also very low immunization coverage which is an important care component of infancy and may 
be attributed to the attitudes and beliefs of the tribal group. According to the World Health Organization (WHO), the definition of health is a state of complete physical, mental and social well-being and not merely the absence of disease or infirmity. The health status of any community is influenced by the interplay of health consciousness of the people, sociocultural, demographic, economic, educational and political factors. The common beliefs, traditional customs, myths, practices related to health and disease in turn influence the health seeking behaviour of autochthonous people (Balgir 2004a). Health is an essential component of the well-being of mankind and is a prerequisite for human development. If general health of an average non-tribal Indian is inferior to the Western and even many Asian counterparts, the health of an average Indian tribal is found to be much poorer compared to the non-tribal counterpart. The health status of tribal populations is very poor and worst of primitive tribes because of the isolation, remoteness and being largely unaffected by the developmental process going on in India.

There is a paucity of comprehensive health research among the tribal populations of India. Most of the studies are isolated and fragmentary in nature. There is an urgent need for initiating the area specific, tribe specific, action oriented health research in consonance with the felt needs of the tribal communities. The research should be mission oriented, having practical applications and directed towards improving the quality of life of tribal people.

Clearly, the challenges for India are multifaceted. In rural areas, reducing child mortality and improving maternal health are major challenges Tribal communities in general and primitive tribal groups in particular are highly disease prone. Also they do not have required access to basic health facilities. They are most exploited, neglected, and highly vulnerable to diseases with high degree of malnutrition, morbidity and mortality (Balgir, 2004). Their misery is compounded by poverty, illiteracy, ignorance of causes of diseases, hostile environment, poor sanitation, lack of safe drinking water and blind beliefs, etc

The situation analysis of health indices of the tribal population in Orissa is worse than the national average: infant mortality rate, 84.2, under five mortality rate, 126.6, children underweight, 55.9, anemia in children, 79.8, children with acute respiratory infection, 22.4, children with recent diarrohea, 21.1, women with anemia, 64.9 per 1000. A high incidence of malnutrition has also been documented in tribal dominated districts of Orissa. This scenario presents a very grim picture about the general health and quality of life of the tribal people in Orissa. There is an urgent need to combat the health problems and take the rehabilitative measures to alleviate the sufferings of the dwindling masses in the country.

\section{Review of literature and information}

A study was done by Pandey and Tiwary to assess the sociocultural and reproductive health practices of primitive tribes of Madhya Pradesh. It collected data from 494 female respondents belonging to the Bharias tribe, 71,277 respondents of the Hill Korwas and 146 respondents from the Kamars respectively. The objective of the study was to collect information on the demographic, sociocultural and health related aspects of the three specified tribes. To collect data on the birth related beliefs and practices, in-depth investigations were carried out in these groups with the help of questionnaires and personal discussions.

A nationwide National Research Health Initiative was carried out in India in the year 2003-2007 coordinated by the Clinical 
Epidemiology Unit, Government Medical College, Nagpur whose goal was to develop operational strategies to improve neonatal survival and decrease their morbidity and mortality in India taking into consideration the existing socio cultural beliefs and practices that influence newborn care in the community.

There are some undesirable new born practices in the community that influence neonatal health care such as withholding early breast feeding, not supplementing with colostrum, application of unhygienic materials and immediate bathing and dressing of baby and administration of prelacteal feeds. Inability to distinguish between severe and non-severe illnesses leads to delay in seeking care and hence neonatal mortality. In addition, there are many other factors like cultural, social, religious and financial that influences the health seeking behaviour of parents.

This nationwide research was launched to promote exclusive breast feeding, eradicate harmful traditional practices, identify inadequate health/medical care, show caretakers how to recognize danger signals in the sick newborn and thus avoid delayed care seeking. The study recommends the need to design behavioral intervention for mothers through strategic and operational modalities aimed to improve health seeking behavior and decision making in caring for newborns.

NHRI is the first population based prospective survey providing information about tribal communities who are understudied and the poorly assessed subgroup of the population facing substantial inequality in healthcare access. Poor infrastructure, lack of human resources and different cultural norms. There is need for socio culturally, contextualized, community based homecare intervention.

Assessment Tools used to assess data were:
Interview schedules of open type for four categories of personnel namely, community stakeholders, medical officers, health workers and NGOs.

Focus Group Discussions five in number by women above forty years having grandchildren, one by husbands/grandfathers, one by women with children less than 6 months, one by MPHW/VHN and the last by birth attendants.

A pilot study was undertaken by Satish Rastogi to diagnose essential needs among scheduled tribes of Nandarbar District of Maharashtra related to education, socio culture and health care needs. The objectives of this study were to:

Take a stock of various common diseases among scheduled tribes of Maharashtra and the steps taken by them as preventive measures.

Study the socio cultural heritage and its positives ethos and values for preservation by the target group.

Examine the socio cultural values as barriers in the way of change among target groups.

To understand educational needs of target group for proposed multimedia regarding awareness of various educational programmes available for them.

Recommend some effective remedial measures for meeting these needs.

The survey adopted a demographic and andragogical approach for assessing the needs of this group. The tool used to assess the essential needs was the Participatory Appraisal Technique. It was conducted for 
two days. On the first day, NGOs, literate tribal youths, educationists, sociologists, and school and college teachers from this tribal group deliberated on the essential needs of the group and common consensus was arrived at. The commoners and illiterates were gathered on the next day and their felt needs assessed and the combination was used to report the results of the study.

Mc Laughin and Braun $\mathrm{K}$ speaking on the strategies to identify details from cultural groups recommend the following strategies be employed:

\section{Learn cultural traditions}

Pay close attention to body language, lack of response or expression of anxiety that may signal that they are in conflict of revelation Ask open ended questions

Remain non judgmental

Salil Basu (2000) on speaking about dimensions of tribal health in India says that the health status of a society is intimately related to its value system, philosophical and cultural tradition and social and economic and political organizations. The health behavior of the individual is closely linked to the way he or she perceives various health problems; what it means and the access to health facilities. The health problems need special attention in the context of tribal communities. The holistic concept of health culture provides a valuable frame work for analyzing the work of anthropologists in health field. However, only a few studies are available in this direction, especially among the tribal population.

\section{Existing problems and shortcomings}

India is second only to China in population. It houses $1 / 7^{\text {th }}$ of the world's population. Out of this, $80 \%$ of India's population lives in the rural area. Every year an estimated 26 million children are born in India which is nearly four million more than the population of Australia. In 2011 , total number of children in the age group 0-6 years is reported as 158.7 million constituting $13 \%$ of the total population. India occupies the $50^{\text {th }}$ position in terms of infant mortality rate. Children, being the "at risk group", need effective care so that they develop to be healthy citizens.

Of the 84 million Scheduled Tribe persons, 38 million are children below 18 years. A majority of the tribal children, about 35 million, live in rural areas. Although the share of scheduled tribes in the population comprises only 8 per cent as per the 2001 Census, with a majority of them inhabiting villages, the share of children is relatively higher among tribal populations (45 per cent) compared to the non-tribal (41 per cent) in India. The location of tribal populations in backward areas, stark inequalities in the availability of basic amenities and the resultant high deprivation levels impact tribal children much more (often this is similar to the scheduled castes/Dalit children) as compared to children belonging to other social groups.

Traditional and cultural practices result in the tribes being treated as the 'other', isolation from mainstream and group identity related behavior, apart from the income poverty is but one element (Thorat, 2008; de Haan, 2007; Das et al., 2010; Gaiha et al., 2008).

Tribal health culture and role of myths, beliefs, culture and tradition on child care practice

Tribal communities are mostly forest dwellers. Their health system and medical knowledge over ages known as 'Traditional Health Care System' depend both on the herbal and the psychosomatic lines of treatment. While plants, flowers, seeds, animals and other naturally available 
substances formed the major basis of treatment, this practice always had a touch of mysticism, supernatural and magic, often resulting in specific magico-religious rites (Balgir, 1997). Faith healing has always been a part of the traditional treatment in the Tribal Health Care System, which can be equated with rapport or confidence building in the modern treatment procedure. Health problems and health practices of tribal communities have been profoundly influenced by the interplay of complex social, cultural, educational, economic and political practices. The study of health culture of tribal communities belonging to the poorest strata of society is highly desirable and essential to determine their access to different health services available in a social set up. Common beliefs, customs, traditions, values and practices connected with health and disease have been closely associated with the treatment of diseases. In most of tribal communities, there is a wealth of folklore associated with health beliefs. Knowledge of folklore of different socio-cultural systems of tribes may have positive impact, which could provide the model for appropriate health and sanitary practices in a given eco-system. The health culture of a community does not change so easily with changes in the access to various health services (Balgir, 2004a). Hence, it is required to change the health services to conform to health culture of tribal communities for optimal utilization of health services. We know that the health comes by evolution, not by revolution. Health must meet the need of the people, as they perceive them. Health cannot be imposed from outside against people's will. It cannot be dispensed to the tribal people. They must want and be prepared to do their share and to cooperate fully in whatever the health programs a community develops (Balgir, 2000a). The problems of shortage of food, poverty, population expansion, malnutrition, health, hygiene and disease burden still persist in
$60 \%$ rural population in India.

The primitive tribes in India have distinct health problems, mainly governed by multidimensional factors such as habitat, difficult terrains, varied ecological niches, illiteracy, poverty, isolation, superstitions and deforestation. The tribal people in India have their own life styles, food habits, beliefs, traditions and socio-cultural activities. The health and nutritional problems of the vast tribal populations are varied because of bewildering diversity in their socio-economic, cultural and ecological settings (Balgir). However, data analysis in view of their ecological, ethnological, cultural and biological diversity is lacking in India and is the need of the hour.

\section{Tribal literacy rate}

Tribal have an illiteracy rate of $47 \%$ with female constituting more than half of the illiterates. The female literacy rate is $14.5 \%$ compared to the general tribal literacy rate of 25.9\%. Education, especially female education, is generally considered a key factor to development. Female education is believed to have a great influence on the maternal and child health as it enhances the knowledge and skills of the mother concerning age at marriage, contraception, nutrition, prevention and treatment of diseases. This also means that the higher infant and child mortality rates among the poorly educated mothers are due to their poor hygienic practices. Moreover, maternal education is related to child health because it reduces the cost of public health related to information on health technology.

As far as child care is concerned, both rural and tribal illiterate mothers are observed to breast-feed their babies. But, most of them adopt harmful practices like discarding of colostrum, giving prelacteal feeds, delayed initiation of breast-feeding and delayed 
introduction of complementary feeds. Vaccination and immunization of infants and children have been inadequate among tribal groups. In addition, extremes of magicoreligious beliefs and taboos tend to aggravate the problems. Only a very few studies are available in this direction, especially among the tribal population. Urgent studies are, therefore, required on different primitive tribal groups of India which are small in size.

\section{In equal health care delivery}

The remoteness of the Tribal settlements and the inaccessibility due to poor connectivity by any means of transport render the tribal group vulnerable to inequities in health care delivery. Therefore it is not uncommon to find a well facilitated and manned health facility in the vicinities of tribal settlements.

Also, lack of incentives to the staff daring to work in these places adds to the in equal health care delivery to the tribes including children. Therefore, the mothers here are deprived of vigilant observation by the health worker who would have otherwise targeted the unsafe child care practices and complemented them with health education.

Almost all literature reviewed by the researcher reveals that there is paucity of research studies on specific tribal groups, their ways of life, practices of child bearing, beliefs underlying rearing practices, superstitions, taboos, faith healing practices, use of herbal remedies for ailments etc.

Considering the increased infant mortality rate reported in the tribal group, their large proportion in our total population, and their right to health and equity, it is necessary to study the beliefs and practices underlying child care.

Since the majority of tribes is illiterate and cannot understand written material, assessment of their beliefs and practices using the conventional questionnaire proves useless. Also data about beliefs are commonly collected using Likert scales, where respondents are asked to rate the strength of agreement or disagreement with a list of belief statements. This method has a number of disadvantages when it is administered to an unknown group. First, there is the danger that respondents may have different interpretations of the language used. Second, Likert scale statements are usually given without context, whereas beliefs tend to be context specific. Third, respondents may be pushed into expressing an opinion even if they have never previously considered the belief stated, thereby giving a misleading overview of their beliefs.

\section{Strategies to improve the situation}

Beliefs are the assumptions we make about ourselves, about others in the world and about how we expect the things to be. Beliefs are about how we think things really are, what we think is really true and what therefore expect as likely consequences that will follow from our behavior. They lead to our practices and any intervention aiming at changing practices should first focus on the foundations of these beliefs with the best possible assessment strategies.

There are similarities as well as differences in attachment beliefs, values and practices amongst parents' from different places and cultures. The infant caregiver attachment relationship includes children's need for responsible parents and parent's desire for securely attached children. It is universal in all people including tribes, the only drawback being that the mothers of the tribal group are yet to cherish motherhood and parenting due to the following reasons:

Most of the tribal mothers are married off at a 
very small age before they understand the responsibilities of motherhood.

Most of the tribal mothers enter into life by force rather than with the excitement of entry into life. Therefore they do not cherish motherhood.

Tribal life sometimes entails illegal relationships without the social custom of marriage and therefore lack of parental responsibility.

Tribal mothers have less choice in family life related to decisions on child care. It is usually from the elder womenfolk of the house or the husband who may not think in the best interest of the child.

Tribes suffer from poverty and all people including females are forced to work for long hours thereby leaving less time for effective parenting. Infants are left to the mercy of kind neighbors or elder siblings who may not satisfy the needs of infants.

Though the above stated reasons contribute to unsafe practices and consequent high mortality, tribes attribute death to the forces of spirits and delve into practices of magic or use of herbs as treatment. The high death rate also adapts them culturally thereby leaving them with no sensitivity or emotion for the deaths. There are traditional healers amongst the tribes who are the popular resort of the community.

Strategies to overcome these shortcomings should be considered in the above mentioned context.

Some suggestions would include:

Evaluate local health traditions in the context of infant mortality. This will help us to critically examine practices. The National
Neonatology Forum of India advises traditional child care practices to be classified as Harmful \& Potentially harmful, Innocuous and Beneficial. NNF also advises to work towards eradication of harmful and potentially harmful practices, not to touch the innocuous ones and to encourage and appreciate beneficial ones.

The Government of India has through the implementation of programmes directed to curtail infant mortality brought in the concept of Accredited Social Health Activists who have a minimum specified qualification and are the locals of the place and therefore have more accessibility and acceptance from the tribes. This group is entrusted with the responsibility of identifying, registering and providing essential maternal and newborn care in the entrusted area with a small incentive under the supervision of Junior Public Health Nurse.

These ASHA workers do not have very less time to devote for assessing home situation, customs, influencing culture and child care practices nor are they efficient enough for analyzing and modifying interventions to suit the situation. It is therefore suggested that the provisions specified under Universal Health Coverage with respect to staffing be implemented with reference to nurses. Professional nurse may be given incentives and family welfare measures to attract them to work in these areas.

\section{Use of Innovation Diffusion Theory}

Everett Rogers defines diffusion as the process by which an innovation is communicated through certain channels over time among the members of a social system. There are four elements in diffusion of innovation process

- Innovation- idea, practice or object 
perceived as new by unit of adoption

- Communication channel-means

of movement of message

- Time-time with which an innovation is adopted by an individual or group

- Social system-a set of interrelated units that are engaged in joint problem solving to accomplish a common goal.

- Effective use of Home Based Nursing Care intervention directly to include promotion of exclusive breast feeding, cessation of practice of prelacteal feeding, provision of proper cord care, warmth, signs, identification of danger signs of illness etc.

- Tackling of other socio economic conditions and providing all the deprivations as identified by. UNICEF.

- Conduction of numerous qualitative researches on tribes, their ways of life etc.

Under the above mentioned circumstances and from various studies quoted above, and from similar studies, it becomes clear that tools for assessment of beliefs and practices on child care in tribal groups should be:

- Interviews that employ open ended questions and

- Focus group Discussions to assess beliefs and practices

- Non Participant Observation to assess practices

- Clinical diaries

\section{Focus group discussion}

A focus group discussion (FGD) is a good way to gather together people from similar backgrounds or experiences to discuss a specific topic of interest. The group of participants is guided by a moderator (or group facilitator) who introduces topics for discussion and helps the group to participate in a lively and natural discussion amongst them. The strength of FGD relies on allowing the participants to agree or disagree with each other so that it provides an insight into how a group thinks about an issue, about the range of opinion and ideas, and the inconsistencies and variation that exists in a particular community in terms of beliefs and their experiences and practices. FGDs can be used to explore the meanings of survey findings that cannot be explained statistically, the range of opinions/views on a topic of interest and to collect a wide variety of local terminology. In bridging research and policy, FGD can be useful in providing an insight into different opinions among different parties involved in the change process, thus enabling the process to be managed more smoothly. It is also a good method to employ prior to designing questionnaires

\section{Detailed outline of process}

FGD sessions need to be prepared carefully through identifying the main objective(s) of the meeting, developing key questions, developing an agenda, and planning how to record the session. The next step is to identify and invite suitable discussion participants; the ideal number is between six and eight.

The crucial element of FGD is the facilitation. Some important points to bear in mind in facilitating FGDs are to ensure even participation, careful wording of the key questions, maintaining a neutral attitude and appearance, and summarizing the session to reflect the opinions evenly and fairly. A detailed report should be prepared after the session is finished. Any observations during the session should be noted and included in the report.

FGDs can also be carried out online. This is particularly useful for overcoming the barrier of distance. While discussion is constrained, the written format can help with reporting on the discussion.

A focus group is not: 
A debate

Group therapy

A conflict resolution session

A problem solving session

An opportunity to collaborate

A promotional opportunity

An educational session

Twelve is the maximum number of questions for any one group. Ten is better, and eight is ideal. Focus group participants won't have a chance to see the questions they are being asked. So, to make sure they understand and carefully respond to the questions posed, questions should be:

Short and to the point

Focused on one dimension each

Unambiguously worded

Open-ended or sentence completion types

Non-threatening or embarrassing

Worded in a way that they cannot be answered with a simple "yes" or "no" answer (use "why" and "how" instead)

There are three types of focus group questions:

Engagement questions: introduce participants to and make them comfortable with the topic of discussion

Exploration questions: get to the meat of the discussion

Exit question: check to see if anything was missed in the discussion

Participant inclusion/exclusion criteria should be established upfront and based on the purpose of the study. Use the criteria as a basis to screen all potential applicants. Focus groups participants can be recruited in any one of a number of ways.

Some of the most popular include:

Nomination - Key individuals nominate people they think would make good participants. Nominees are familiar with the topic, known for their ability to respectfully share their opinions, and willing to volunteer about 2 hours of their time.

Random selection - If participants will come from a large but defined group (e.g. an entire high school) with many eager participants, names can be randomly drawn from a hat until the desired number of verified participants is achieved.

- All members of the same group Sometimes an already existing group serves as an ideal pool from which to invite participants

- Same role/job title - Depending on the topic, the pool might be defined by position, title or condition (e.g., community health nurses).

- Volunteers - When selection criteria is broad, participants can be recruited with flyers and newspaper ads

Once a group of viable recruits has been established, call each one to confirm interest and availability. Give them times and locations of the focus groups. Organize the times, locations and people involved for all the groups you have scheduled and secure verbal confirmation. Tell participants that the focus group will take about one and half to two hours. Give them a starting time that is 15 minutes prior to the actual start of the focus group to have a bite to eat, and settling in to the group. Arrange for a comfortable room in a convenient location.

Depending on your group, you may also what to consider proximity to a bus line. The room should have a door for privacy and table and chairs to seat a circle of up to 12 people (10participants and the moderator and assistant moderator). Many public agencies (churches, libraries) have free rooms available. Arrange for food. At a minimum, 
offer a beverage and light snack. It is OK to offer a full meal but be sure to add an additional 30 to 45 minutes to the entire process so that everyone can finish eating before the group begins.

\section{Semi-structured interview (SSI)}

Purpose: To gain information from an individual or small group on an issue.

Description: Semi-structured interviews are guided conversations where broad questions are asked, which do not constrain the conversation, and new questions are allowed to arise as a result of the discussion. This is different from questionnaires and surveys where there are very structured questions that are not deviated from. A semi-structured interview is therefore a relatively informal, relaxed discussion based around a predetermined topic.

A semi-structured interview is a PRA method that engages villagers in a conversation through a series of guide questions (not structured questionnaire) relevant to the villagers. Important information is generated by talking with villagers about topics that interest them. SSI can be used with individuals, key informants, interest groups or other small groups of villagers (i.e. women's groups). It is usually best to conduct such interviews in pairs with the person doing the interview and one taking detailed notes. The process of a semi-structured interview involves the interviewer presenting the context of the study and its objectives to the interviewee or interview group (such as a family or household). The set of questions are prepared but open, allowing the interviewees to express opinions through discussion. Questions are generally simple, with a logical sequence to help the discussion flow. Interview questions should be tested prior to interviews.
Training people to conduct a semi-structured interview is important and practice is required to become an effective interviewer. Training needs to address team preparation, interview context, sensitive listening, sensitive questioning, judging responses, recording the interview and self-critical review (Pretty et al., 1995).

It involves the preparation of an interview guide that lists a pre-determined set of questions or issues that are to be explored during an interview. This guide serves as a checklist during the interview and ensures that basically the same information is obtained from a number of people. Yet, there is a great deal of flexibility. The order and the actual working of the questions is not determined in advance. Moreover, within the list of topic or subject areas, the interviewer is free to pursue certain questions in greater depth.

The advantage of the interview guide approach is that it makes interviewing of a number of different persons more systematic and comprehensive by delimiting the issues to be taken up in the interview. Logical gaps in the data collected can be anticipated and closed, while the interviews remain fairly conversational and situational.

The weakness of this approach is that it does not permit the interviewer to pursue topics or issues of interest that were not anticipated when the interview guide was elaborated. Also, interviewer flexibility in wording and sequencing questions may result in substantially different responses from different persons, thus reducing comparability.

Procedures in using a semi-structure interview (SSI)

Prepare a checklist of topics and guide 
questions for discussion and record these in a notebook.

Select individuals, key informants, interest groups, or other small groups of villagers to interview. Get a good representation of the villagers- spatial, gender, wealth class, etc.

Observe proper interviewing techniques.

Use the checklist of topics and guide questions (see sample) but allow flexibility in the conversation so that issues can be explored as they arise.

Probe (use relevant follow up questions as needed).

Ask questions that are relevant to the villagers being interviewed (individual or group).

Use open-ended questions (not answerable by yes or no).

Record the important points in each interview in a notebook.

Modify the checklist of topics and guide questions as new issues arise during the conversation.

\section{Structured Direct Observation}

What people say and what they do may be two different things. Sometimes people idealize a situation and tell things which are more a description of how things should be than how things are. Other reasons for this difference are that while talking about routine activities a person is only able to give information about some aspects of this activity. One reason for this is that these activities are often routines and done without reflection and they have been learned by observation and by doing. Usually farmers do not talk much about their routine activities, like selection of seeds, preparation of seed mixtures etc., they simply do it. Farmers, like others, often say things, which do not correspond with reality. What people tell in interviews is not a picture of the reality, but their mental construction of the activities. Direct observation allows a cross-check of findings of what people tell. Direct observation can also be used to generate onthe-spot questions in direct interaction with the farmers. This helps farmers to explain things which are grounded in their activity and not on their remembrance of the activity. On the other hand it helps scientists to generate questions. Structured direct observation is better conducted by a team to minimize individual biases. Always the observation has to be discussed with the people to gain an insider's perspective.

These are the various approaches to assess child care beliefs and practices in tribal groups.

\section{Nursing Implications}

Let us now look into the various implications involved in the assessment of beliefs and practices of child care practices in tribal groups. The implications may broadly be classified under:

Nursing Administration

Nursing Education

Nursing Service

Nursing Research

\section{Nursing administration}

Nurses at the policy making level can interact with Government and Non governmental agencies in planning and participating in interventions related to identification, creating awareness and effecting behavioral changes among tribal groups so that mortality and morbidity indicators are brought down. Also Nursing leaders can lobby and influence the Government to create new roles like Social Physicians or Community Nurse Practitioners who will work in close collaboration with the tribal groups. 


\section{Nursing education}

As teachers, nurses can instill empathetic thinking in the minds of student nurses regarding ethnic groups so that health is protected while culture is also preserved. Among those willing for transition, the teacher with her student can, through guided steps take them through the path of change.

\section{Nursing service}

In the clinical area, nurses confront patient from different cultural groups including tribes. Here nurses have the golden opportunity to assess beliefs as well as to observe child care practices. The nurse in the hospital has more influence and acknowledgement from the public and their words of care are better accepted.

Table.1 State/Union Territory wise Number of Tribal Groups in India ${ }^{2}$

\begin{tabular}{|c|c|c|}
\hline SI.No & State/UT & No. of Tribal Group \\
\hline 1. & Andhra Pradesh & 35 \\
\hline 2. & Arunachal Pradesh & 16 \\
\hline 3. & Assam & 15 \\
\hline 4. & Bihar & 33 \\
\hline 5. & Chhattisgarh & 42 \\
\hline 6. & Goa & 8 \\
\hline 7 & Gujarat & 32 \\
\hline 8. & Himachal Pradesh & 10 \\
\hline 9. & Jammu \& Kashmir & 12 \\
\hline 10. & Jharkhand & 32 \\
\hline 11. & Karnataka & 50 \\
\hline 12. & Kerala & 43 \\
\hline 13. & Madhya Pradesh & 46 \\
\hline 14. & Maharashtra & 47 \\
\hline 15. & Manipur & 34 \\
\hline 16. & Meghalaya & 17 \\
\hline 17. & Mizoram & 15 \\
\hline 18. & Nagaland & 5 \\
\hline 19. & Orissa & 62 \\
\hline 20. & Rajasthan & 12 \\
\hline 21. & Sikkim & 4 \\
\hline 22. & Tamil Nadu & 36 \\
\hline 23. & Tripura & 19 \\
\hline 24. & Uttarakhand & 5 \\
\hline 25. & Uttar Pradesh & 15 \\
\hline 26. & West Bengal & 40 \\
\hline 27. & Andaman \& Nicobar Islands & 6 \\
\hline 28. & Dadra \& Nagar Haveli & 7 \\
\hline 29. & Daman \& Diu & 5 \\
\hline 30. & Lakshadweep & All inhabitants. \\
\hline
\end{tabular}


Table.2 Key Health Indicators Among Tribes (NFHS 2005-06)

\begin{tabular}{|l|l|l|}
\hline Indicators & Scheduled Tribes & Total \\
\hline Infant Mortality & 62.1 & 57 \\
\hline Neo-natal Mortality & 39.9 & 39 \\
\hline Post-natal Mortality & 22.3 & 18 \\
\hline Child Mortality & $\mathbf{3 5 . 8}$ & 18.4 \\
\hline Under Five Mortality & 95.7 & 74.3 \\
\hline ANC Checkup & 70.5 & 77.1 \\
\hline \% Institution Delivery & $\mathbf{1 7 . 7}$ & 38.7 \\
\hline Childhood Vaccination & $\mathbf{3 1 . 3}$ & 45.6 \\
\hline
\end{tabular}

\section{Nursing research}

Literature points out to the paucity of studies on tribal groups more so by nurses. Tribal area offers more opportunities and challenges for the nurse researcher since a lot can be accomplished through mixed method research, combining qualitative and quantitative approaches.

In conclusion, beliefs should be based on scientific rationale. It is the lack of this awareness that results in faulty care practices. Any community belief is held as superior by members of that group. It is necessary to identify unsafe practices, do away with them without harming the ethnicity of the group providing them culturally sensitive care leaving them with a feeling of community acceptance and not a sense of threat.

\section{References}

Balgir, R.S. "Tribal Health Problems, Disease Burden and Ameliorative Challenges in Tribal Communities with special emphasis on Tribes of Orissa" - Proceedings of National Symposium on Tribal Health, www.tribal.nic.in

G. D. Pandey, R.S. Tiwary, "Sociocultural Reproductive Health Practices of Primitive Tribes of Madhya Pradesh: Some Observations", The Journal of
Family Welfare, 47(2):Oct.2001:2733.

Report on NCLEN's "National Neonatal Health Research Initiative" coordinated by Clinical Epidemiology Unit, Government Medical College, Nagpur, 2003.

Satish Rastogi, " Educational, Sociocultural and Health Care Needs among Scheduled Tribes of Nandurbar District, Maharashtra: A Diagnosis" VCM Open University Nasik, India

Mc Laughin L, Braunk K “Asian and Pacific Islander Cultural Values: Considerations for Health Care Decision Making" Health and Social Work, 23(2): 1998, 116-126.

Salil Basu, "Dimensions of Tribal Health in India" Health and PopulationPerspectives and Issues, 23(2):2000, 61-70.

Family Welfare Statistics in India, Ministry of Health and Family Welfare, Government of India, 2013.

Preet Rustagi et al.,, "Scheduled Tribe Children in India: Multiple Deprivations and Locational Disadvantage", IHD-UNICEF Working Paper Series, Children of India: Rights and Opportunities, 2011. www.social researchmethods.net.

Amy J. Kesterton, John Cleland, "Neonatal Care in Rural Karnataka: Healthy and Harmful Practices: The Potential for 
Change" BioMed Central Pregnancy and Childbirth, 2009; 9(20): 9-20.

http://www.ucc.ie/hfrg/projects/respect/urmet hods/focus.htm

Universita 'Degli Studi Di Padora Fa ol ta 'Di Agraria, Participatory Rural Appraisal- Concepts, Methodologies and Techniques, 2003; Pp. 16, 25. www.tribal.nic.in

www.ker.gov.in

Kerala Institute for Research Training and Development Studies for SC/ST, Government of Kerala

www.ncm.nic.in

www.socialresearchmethods.net

\section{How to cite this article:}

Rathi, M. 2019. Assessment of Childcare Beliefs and Practices in Tribal Population. Int.J.Curr.Microbiol.App.Sci. 8(03): 1639-1653. doi: https://doi.org/10.20546/ijcmas.2019.803.191 